Sharif University of Technology
Scientia Iranica
Transactions E: Industrial Engineering
wCIENTIA

\title{
Modeling of location-distribution considering customers with different priorities by a lexicographic approach
}

\author{
M. Bashiri* and H. Hasanzadeh \\ Faculty of Engineering, Department of Industrial Engineering, Shahed University, Tehran, Iran.
}

Received 22 June 2013; received in revised form 28 October 2014; accepted 4 May 2015

\section{KEYWORDS}

Distribution network

design;

Customer priority;

Lexicography;

Mixed integer

programing.

\begin{abstract}
In this paper, a multi-echelon location-distribution problem is modeled considering customer priorities. A lexicographic approach is implemented to determine the most preferred distribution path according to the priorities of customers. The predetermined number of trucks moving from depots and satellites is considered in the proposed model. The results show that the proposed approach can better consider the customers with different priorities, while more important customers will have low total costs compared to the classical approach. Moreover, the sensitivity analysis has been done for discovering the effects of related parameters in the model.
\end{abstract}

(C) 2016 Sharif University of Technology. All rights reserved.

\section{Introduction}

A supply chain links all entities of the supply chain, from the supplying of raw materials to the final product delivery, as mentioned by Noorul and Kannan [1]. A supply chain can be configured by network design decisions, which has a significant impact on logistics costs and responsiveness [2]. Distribution Network Design (DND) is a well-known problem of supply chain network design, which considers the location of central depots, depots, satellites, and their allocation structure to the customers. DND problems involve two types; in the first type, flows of products are optimized according to an existing distribution network, and in the second one, the best configuration of distribution centers and facilities in the network is determined according to total costs [3]. The presented formulation in this paper is based on the second type of DND problems.

There are several strategic decisions for DND problems, including facility location, transportation,

\footnotetext{
*. Corresponding author. Tel: +98 2151212092 ;

Fax: +982151212020

E-mail addresses: Bashiri.m@gmail.com (M. Bashiri);

Hasanzadeh.ie@gmail.com (H.Hasanzadeh)
}

inventory, and quality of customer service level [4]. Moreover, there are many production factors in the real world, which can be taken into account in DND problems, such as the number of echelons, facility capacity, number of products, time periods, stochastic demands, single or multiple sourcing, routing, and etc. However, considering these concepts can simultaneously increase model complexity. So, many studies in the literature have considered some of the mentioned factors [5]. In this study, a mathematical formulation of multi-echelon DND problem is presented considering customers' priorities and network capacity. Since the main difference between the proposed and the typical models in DND Problem is in giving priority to customers in determining location of depots and satellites, some of the reasons for prioritizing customers are described in the following:

1. Customers credit: Defined as categorizing customers based on their historical behavior; for example, IBM Company implemented a part stocking plan to support a time-based service strategy and wanted to divide services level for three sets of customers according to their credit [6];

2. Service level: Many companies consider service time 
and their services are proportional with waiting time. For example, postal services can be divided into two levels (ordinary and express), and determining location of post offices according to the service type would be more reasonable;

3. Emergency condition: Distribution centers can be located based on the risk level of vulnerable points (demand points in normal distribution), while vulnerable points have been categorized based on risk levels. For example, Mclay and Mayorga [7] presented a model in Emergency Medical Service (EMS) considering customer priorities and suggested Markov decision process model to determine optimal dispatching policies for prioritized customers.

Generally, customer service level in DND problems is discussed for all customers; Melachrinoudis et al. [8] and Chan et al. [9] used multi-objective to minimize costs and increase service level of customers. Moreover, there are several studies in other fields of study that consist of increasing service level of special customers. For example, recently, Sapna Isotupa and Samanta [10] described a model considering two kinds of customers (with high and low priorities) in inventory system. A similar work was introduced in the supply chain management by Tempelmeier and Horst [11]. To our knowledge, there is not any study to configure a distribution network based on the customers' priorities.

On the other hand, priorities of customers can be determined by some of the decision-making tools; for example, Barbarosoglu and Yazgac [12] determined customers' priorities using Analytic Hierarchy Process (AHP) method; Khaji and Shafaei [13] used a Fuzzy Analytic Network Process (FANP) to calculate customers' priorities in a supply chain network design.

This paper has been organized as follows. In the next section, the proposed model is presented containing related parameters, variables, assumptions, and mathematical formulation. Solution approaches based on customers' priorities are presented in Section 3. Section 4 contains some hypothetical numerical examples. Moreover, the sensitivity analyses are reported in Section 5. Finally, the conclusion is described in the last section.

\section{The proposed model}

In this section, a new mathematical model is proposed based on the developed model by Gendron et al. [14]. In their research, two kinds of formulations, including arcbased and path-based, are generated. The distinction between arc-based and path-based formulations is in using decision variables for determining distribution paths from suppliers to customers among different echelons that are illustrated in Figure 1. Each of these

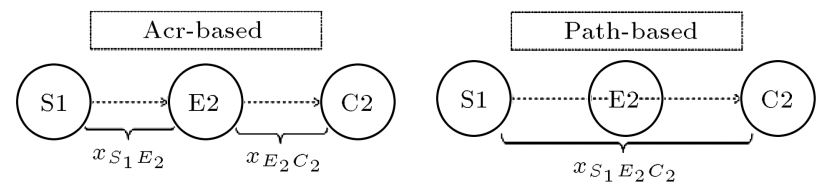

Figure 1. Illustration of arc-based and path-based formulations.

two formulations has its advantages and disadvantages. For example, adding a new echelon between suppliers and customers in the arc-based formulation does not change decision variables, except for the added echelon; however, all of the flow decision variables are changed in the path-based formulation by adding new subscript, because of adding the new echelon [15]. Moreover, the number of variables in the arc-based formulation is less than that of path-based formulation (suppose that there are 3 suppliers (S), 3 satellites (E), and 4 customers (C); so, there are 21 flow decision variables including $x_{S E}$ and $x_{E C}$ in the arc-based formulation; while there are 36 variables including $x_{S E C}$ in the pathbased formulation). However, Gendron and Semet [14] showed that linear programming relaxation of the path-based formulation provides a better lower bound than that of arc-based formulation. It is worth to mention that when there is no satellite in a problem, the arc-based and path-based formulations are equivalent.

As mentioned before, the arc-based formulation has more flexibility in some changing compared to the path-based formulation; so, it has been considered to develop a new model in this study. Furthermore, since customers' priorities and number of iterations are determined by adding new subscripts to variables in the proposed model, we try to use the formulation with less subscript.

The main goals of the proposed model in this study are to find the best location of depots and satellites, to determine an optimal distribution center network, and to allocate customers to opened depots and satellites, according to the related costs considering priorities of customers. In the presented model, a limited number of available trucks are considered in design of the distribution network.

The proposed mathematical model is based on the following assumptions:

1. There are two types of large- and small-size trucks to move between the central depot, and depots and between depots and satellites, respectively;

2. Customer priorities are obtained according to the designer preferences; so, the model should be solved according to customers' ordinal preferences;

3. There are single allocations in the allocation of customers to satellites, and allocation of satellites to depots; 
4. There is a limited predetermined number of trucks in depots and satellites.

\subsection{Definitions}

Since the proposed model is based on the lexicographic approach and should be solved iteratively according to the customers' priorities, so some parameters of the model should be updated in solving a problem for the customer with the $t$ th priority. For example, the number of available trucks in depots and satellites in each of the iterations depends on the result of the pervious iterations that is computed according to the difference between the number of available trucks in the current iteration and the number of used trucks in the previous iterations. On the other hands, there are some variables such as location of depots and satellites that are affected by the results of the pervious iteration. For example, if some depot is opened in each iteration, it should be considered open in the next iterations and adding of their establishing costs should be avoided. So, the proposed model is formulated for updating parameters and variables in different iterations by updating iteration counter or customer priority $(t)$.

Generally, variables of the proposed model can be categorized based on three aspects including: I) type of variables (binary, integer, and continuous variables), II) dependency or independency on the results of pervious iterations (some decision variables should be updated in each iteration, which are called dependent variables), III) auxiliary or main decision variables. Parameters, variables, objective functions, and constraints are presented as follow:

\section{Parameters}

$T \quad$ Number of customer priorities; $t$ is its counter. Iteration $t$ in the model means the customer with $t$ th priority;

$D \quad$ Set of potential sites to locate depots;

$S \quad$ Set of potential sites to locate satellites;

$L \quad$ Set of customers;

$L^{t} \quad$ Set of customers with priority $t$;

$D_{j}^{s} \quad$ Set of potential sites to locate depots connected to satellite $j \in S$;

$S_{i}^{D} \quad$ Set of potential sites to locate satellites connected to depot site $i \in D$;

$S_{l}^{L} \quad$ Set of potential sites to locate satellites connected to customer $l \in L$;

$S^{L t} \quad$ Set of potential sites to locate satellites connected to customers with priority $t$;

$L t_{i}^{D} \quad$ Set of customers with priority $t$ connected to depot $i \in D$;

$L t_{j}^{S} \quad$ Set of customers with priority $t$ connected to satellite $j \in S$; $n_{l} \quad$ Number of product units that should be delivered to customer $l \in L$;

$Q \quad$ Number of product units of one pack;

$P \quad$ Capacity of large-size trucks to transport product units;

$R \quad$ Capacity of small-size trucks to transport product units;

$f_{i} \quad$ Fixed establishment cost of depot $i \in D$;

$f s_{j} \quad$ Fixed establishment cost of satellite $j \in S$;

$g_{j} \quad$ Packing cost per one pack in the satellite $j \in S$;

$d_{i} \quad$ Transportation cost of large-size truck to transport product units from center depot to depot $i \in D$;

$e_{i j} \quad$ Transportation cost of small-size truck from depot $i \in D$ to satellite $j \in S_{i}^{D}$;

$c_{j l} \quad$ Transportation cost between satellite $j \in S$ and customer $l \in L_{j}^{S}$;

$T c_{c}^{t} \quad$ Number of available large-size trucks in center depot for solving the model in the $t$ th iteration;

$T c_{i}^{t} \quad$ Number of available small-size trucks in depot $i \in D$ for solving the model in the $t$ th iteration.

The last two parameters should be obtained according to the results of the previous iteration.

\section{Main decision variables}

Location of depots and satellites, allocation of customers to satellites and satellites to depots, the number of needed trucks in depots and satellites, the number of product packs in satellites, and the volume of transferred products from depots are considered to be the main decision variables in this paper. Note that all of them are independent and do not need to be updated for different customer priorities.

\section{Binary variables}

$Y_{i}=1 \quad$ If depot $i$ is established; 0 otherwise;

$Y s_{j}=1 \quad$ If satellite $j$ is established; 0 otherwise;

$X_{j l}=1 \quad$ If customer $l$ is allocated to the satellite $j$; 0 otherwise;

$W_{i j}=1 \quad$ If satellite $j$ is allocated to the depot $i$; 0 otherwise.

\section{Integer variables}

$u_{j} \quad$ Number of required product packs in satellite $j$;

$t_{i} \quad$ Number of large-size trucks needed in depot $i$; 
$h_{i j} \quad$ Number of small-size trucks needed to transport products from depot $i$ to satellite $j$.

\section{Continuous variables}

$V_{i j} \quad$ Product volume transshipped from depot $i$ to satellite $j$.

\section{Auxiliary decision variables}

As mentioned before, the proposed model should be solved iteratively and some determined decision variables in each iteration, such as location of depots and satellites, should be considered in the next iterations; so, the result of the pervious iterations is regarded by defining some auxiliary variables in different iterations. Moreover, the number of extra needed trucks is determined by the difference between the number of needed trucks and the number of used trucks (considering the number of available trucks) in depots and satellites.

\section{Dependent binary variables}

$K y_{i}^{t}=1 \quad$ If depot $i$ has been established in the previous iterations of $1 . . t-1 ; 0$ otherwise;

$O_{j}^{t}=1 \quad$ If satellite $j$ has been established in the previous iterations of $1 . . t-1 ; 0$ otherwise.

\section{Dependent continuous variables}

$F_{i j}^{t} \quad$ Unoccupied capacity of the used trucks from depot $i$ to satellite $j$ in iteration $t$

$V T_{i}^{t} \quad$ Unoccupied capacity of the used trucks from the center depot to depot $i$ in iteration $t$;

$s s_{j}^{t} \quad$ Unoccupied capacity of the product packs in satellite $j$ in iteration $t$.

\section{Independent integer variables}

$N_{i} \quad$ Number of large-size trucks used in depot $i$;

$N s_{i j} \quad$ Number of small-size trucks used to transport products from depot $i$ to satellite $j$.

\subsection{Mathematical model}

In this section, we formulate a two-echelon distribution network that can be used to determine the location of depots and satellites based on the priorities of customer. Objective function of the proposed model involves three types of cost including: establishment, operational, and transportation costs. The proposed model should be solved iteratively according to the customers' priorities and establishment costs should be considered only one time when is being established for the first time. So, some auxiliary variables, including $k y_{i}^{t}$ and $o_{j}^{t}$, are used to determine established depots and satellites in the previous iterations and establishment cost of each depot or satellite can be considered when the corresponding auxiliary variables are equal to zero. Since operational costs are related to packing products in the satellites, they are computed based on the number of required product packs in each satellite $\left(g_{j} u_{j}^{t}\right)$. Finally, transportation costs are computed according to the number of used trucks in the depots and satellites, and the number of products that should be transferred between satellites, and customers. It is worth to mention that since the number of trucks and their capacity are limited, surplus costs should be considered when the number of needed trucks are more than that of the available trucks. In this study, we have multiplied the transportation cost (the trucks sweep) by 2 for transferring surplus products. The objective function and constraints are presented as follows:

$$
\begin{aligned}
\min & \sum_{i \in D} f_{i} y_{i}^{t}\left(1-k y_{i}^{t}\right)+\sum_{j \in S} f s_{j} y s_{j}^{t}\left(1-o_{j}^{t}\right) \\
& +\sum_{j \in S} g_{j} u_{j}^{t}+\sum_{i \in D} d_{i} N_{i}+\sum_{i \in D} \sum_{j \in S} e_{i j} N s_{i j} \\
& +\sum_{j \in S} \sum_{l \in L} c_{j l} n_{l} x_{j l}+\sum_{i \in D} 2 d_{i}\left(t_{i}-N_{i}\right) \\
& +\sum_{i \in D} \sum_{j \in S} 2 e_{i j}\left(h_{i j}-N s_{i j}\right)
\end{aligned}
$$

Subject to:

$$
\begin{aligned}
& \sum_{j \in S_{l}^{L t}} x_{j l}=1 \quad \forall l \in L t, \\
& \sum_{i \in D_{j}^{S}} w_{i j} \leq 1 \quad \forall j \in S^{L t}, \\
& N_{i} \leq t_{i} \quad \forall i \in D, \\
& N_{i} \leq T c_{c}^{t} \quad \forall i \in D, \\
& N s_{i j} \leq h_{i j} \quad \forall i \in D, \quad \forall j \in S, \\
& N s_{i j} \leq T c_{i}^{t} \quad \forall i \in D, \quad \forall j \in S, \\
& w_{i j} \leq y_{i}^{t} \quad \forall i \in D, \quad \forall j \in S_{i}^{D}, \\
& w_{i j} \leq y s_{j}^{t} \quad \forall i \in D, \quad \forall j \in S_{i}^{D}, \\
& \sum_{j \in S_{i}^{D}} v_{i j} \leq\left(\sum_{l \in L t_{i}^{D}} n_{l}\right) y_{i}^{t} \quad \forall i \in D, \\
& \sum_{j \in D_{j}^{S^{L t}}} v_{i j} \leq \sum_{l \in L_{j}^{S^{L t}}} n_{l} x_{j l} \quad \forall j \in S^{L t},
\end{aligned}
$$




$$
\begin{aligned}
& v_{i j} \leq\left(\sum_{l \in L_{j}^{S t}} n_{l}\right) w_{i j} \quad \forall i \in D, \quad \forall j \in S^{L t} \\
& x_{j l} \leq \sum_{i \in D_{j}^{S}} w_{i j} \quad \forall j \in S^{L t}, \quad \forall l \in L t_{j}^{s}, \\
& \sum_{l \in l t_{j}^{S}} n_{l t} x_{j l} \leq u_{j}^{t} Q+s s_{j}^{t} \quad \forall j \in S, \\
& \sum_{j \in S_{i}^{D}} v_{i j} \leq t_{i} P+V T_{i}^{t} \quad \forall i \in D \\
& v_{i j} \leq h_{i j} R+F_{i j}^{t} \quad \forall i \in D, \quad \forall j \in S_{i}^{D}, \\
& k y_{i}^{t+1} \leq y_{i}^{t}+k y_{i}^{t} \quad \forall i \in D \\
& o_{j}^{t+1} \leq y s_{j}^{t}+o_{j}^{t} \quad \forall j \in S, \\
& s s_{j}^{t+1}=\left(u_{j}^{t} Q-\sum_{l \in L t_{j}^{S}} n_{l t} x_{j l}\right)+s s_{j}^{t} \quad \forall j \in S, \\
& V T_{i}^{t+1}=\left(t_{i} P-\sum_{j \in S_{i}^{D}} v_{i j}\right)+V T_{i}^{t} \quad \forall i \in D \\
& F_{i j}^{t+1}=\left(h_{i j} R-v_{i j}\right)+F_{i j}^{t} \quad \forall i \in D, \quad \forall j \in S, \\
& T c_{c}^{t+1}=T c_{c}^{t}-\sum_{i \in D} N_{i} \\
& T c_{i}^{t+1}=T_{c_{i}}^{t}-\sum_{j \in S} N s_{i j} \quad \forall i \in D, \\
& u_{j}, t_{i}, h_{i, j}, N_{i}, N s_{i, j} \geq 0 \text { and integer } \\
& x_{j l}, w_{i j}, y_{i}, k y_{j}, y s_{j}, o_{j} \in\{0,1\}, \\
& v_{i j}, F_{i, j}, V T_{i}, s s_{j} \geq 0 .
\end{aligned}
$$

Eq. (1) is the objective function, composed of eight terms. Establishment costs of depot and satellites in each iteration are considered in the first two terms. The third term represents operational costs in satellites. Transportation costs between the central depot and depots, depots and satellites, satellites and customers are computed in the next terms, respectively. Transportation costs of surplus products between central depot and depots and depots and satellites are considered in the last two terms. Constraint (2) ensures that each customer is allocated to only a single satellite. Constraint (3) ensures that each satellite is allocated to one depot. Constraints (4) and (5) show that the number of used trucks to transport products from the central depot to depot $i$ should be less than the number of needed trucks for this depot and the maximum number of existing trucks, respectively. Constraints (6) and (7) show that the number of used trucks to transport products from depot $i$ to satellite $j$ should be less than the number of needed trucks for corresponding satellite and the maximum number of existing trucks in depot $i$, respectively. Constraints (8) and (9) guarantee that the link between $i$ and $j$ can be activated when both depot $i$ and satellite $j$ are established. Constraints (10) to (13) determine the number of products that should be transshipped. Constraints (14) to (16) determine the number of required trucks in depots and satellites and also the number of batches, respectively. Constraints (17) and (18) determine the established depots and satellites for the next iteration considering current iteration solution. Constraints (19) to (21) determine the unoccupied capacity of used trucks and unoccupied capacity of packs for the next iteration, which are related to Constraints (14) to (16). Constraints (22) and (23) determine the number of available trucks for the next iteration.

\subsection{Model linearization}

The proposed mathematical model is nonlinear at the present form due to the first two statements of objective function. The linearization scheme is based on the introduced method of [16], so a new variable is introduced as $A_{i}^{t}$ instead of multiplication of two binary variables:

$$
A_{i}^{t}=\left(y_{i}^{t}\right)\left(k y_{i}^{t}\right) \quad \forall i \in D .
$$

By the mentioned replacement, the following additional constraints should be added to the proposed model:

$$
\begin{aligned}
& y_{i}^{t}+k y_{i}^{t} \leq A_{i}^{t}+1 \quad \forall i \in D, \\
& y_{i}^{t}+k y_{i}^{t} \geq 2 A_{i}^{t} \quad \forall i \in D, \\
& A_{i}^{t} \geq 0 \quad \forall i \in D .
\end{aligned}
$$

Constraint (40) ensures that when $y_{i}^{t}=1$ and $k y_{i}^{t}=$ 1, $A_{i}^{t}$ must be 1; Constraint (41) ensures that when $y_{i}^{t}=0$ and $k y_{i}^{t}=0, A_{i}^{t}$ must be 0 . In spite of the determined variable type for $A_{i}^{t}$, it will get a binary value because of other existing Constraints (40) and (41). Similarly, by introducing new variable $B_{j}^{t}$ instead of $\left(o_{j}^{t}\right)\left(y s_{j}^{t}\right)$, Constraints (43) to (45) should be added to the proposed model:

$$
\begin{aligned}
& y s_{j}^{t}+o_{j}^{t} \leq B_{j}^{t}+1 \quad \forall j \in S, \\
& y s_{j}^{t}+o_{j}^{t} \geq 2 B_{j}^{t} \quad \forall j \in S, \\
& B_{j}^{t} \geq 0 \quad \forall j \in S .
\end{aligned}
$$


So, the proposed linear model can be stated as follows:

$$
\begin{aligned}
& \sum_{i \in D} f_{i} y_{i}^{t}-\sum_{i \in D} f_{i} A_{i}^{t}+\sum_{j \in S} f s_{j} y s_{j}^{t}-\sum_{j \in S} f s_{j} B_{j}^{t} \\
& +\sum_{j \in S} g_{j} u_{j}^{t}+j^{t}+\sum_{i \in D} d_{i} N_{i}+\sum_{i \in D} \sum_{j \in S} e_{i j} N s_{i j} \\
& +\sum_{j \in S} \sum_{l \in L} c_{j l} n_{l} x_{j l}+\sum_{i \in D} 2 d_{i}\left(t_{i}-N_{i}\right) \\
& +\sum_{i \in D} \sum_{j \in S} 2 e_{i j}\left(h_{i j}-N s_{i j}\right)
\end{aligned}
$$

subjected to Constraints (2) to (38), and also (40) to (45).

\section{Solution approaches according to customer priorities}

From the point of view of cost, four approaches are proposed, which consider the interaction between the priorities customers and the total cost. In fact, if the priority is more important, then despite the increase in the total system cost, the designer prefers to decrease high priority customers-related cost as a main objective. However, the importance of customers and total cost depends on the nature of the problem; so, in this study, four approaches have been proposed with variable attention to the total cost and customer priority.

In the first approach, the designer considers the total cost of the system without regarding the importance of the priorities of customers (Classical approach). In the second one, the designer only considers priorities of customers even if total cost increases
(Approach 1). In the next proposed approach (Approach 2), both total cost and priorities of customers are considered, but more attention is paid customers' priorities. In the last approach (Approach 3), it is tried to have a network with consideration of total cost and customers' priorities with the same importance. Each proposed approach will be introduced in the following sub-sections.

\subsection{Approach 1 (just attention to the customers' priorities )}

In this approach, priority of customer is an essential element, which should be considered strictly. Sometimes, in order to satisfy demands of a customer with lower priority, we may have to establish new depots or satellites, which increase the total cost. The flowchart of Approach 1 is depicted in Figure 2. In this proposed approach, the previous established depots or satellites will be remained and the network can be equipped by establishing new facilities for satisfying demands of customers with lower priorities.

\subsection{Approach 2 (more attention to the priorities of customers)}

In this approach, more attention is given to the priority of customers. Actually, after solving model 1, some depots and satellites are established. Then, the cost of established depots and satellites is considered to be zero; and the model, considering the classical approach, is solved again. By this replication, the optimal paths may be changed. The flowchart of this approach is illustrated by Figure 3 . In fact, this approach is an improved version of the previous approach, and it will try to minimize the total transportation cost by considering opened facilities as model inputs.

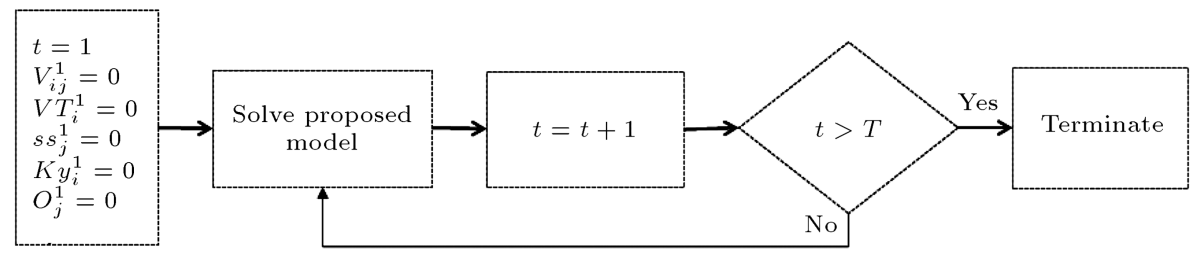

Figure 2. Flowchart of Approach 1.

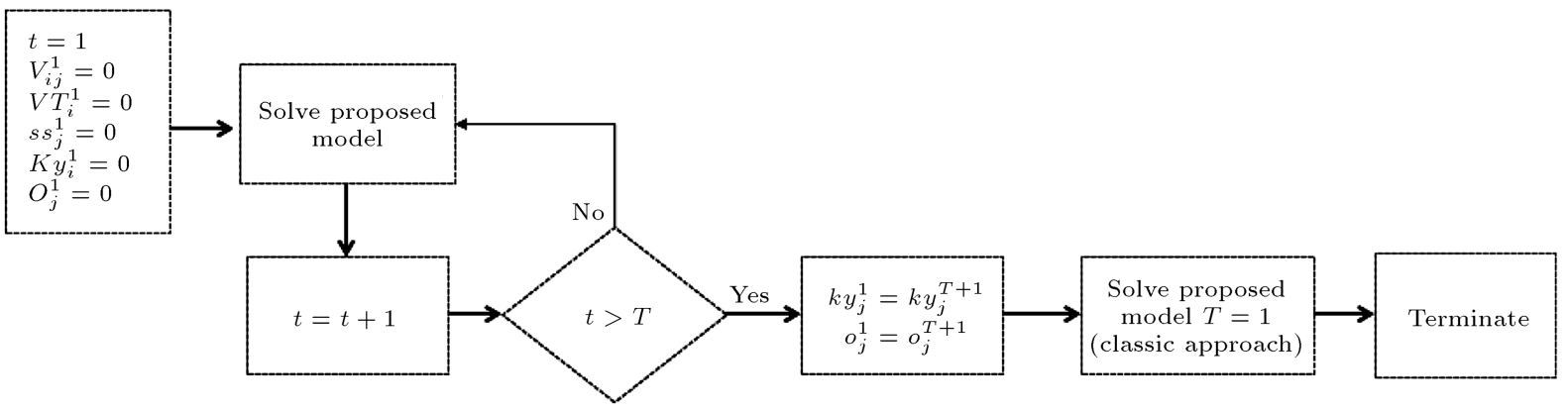

Figure 3. Flowchart of Approach 2. 


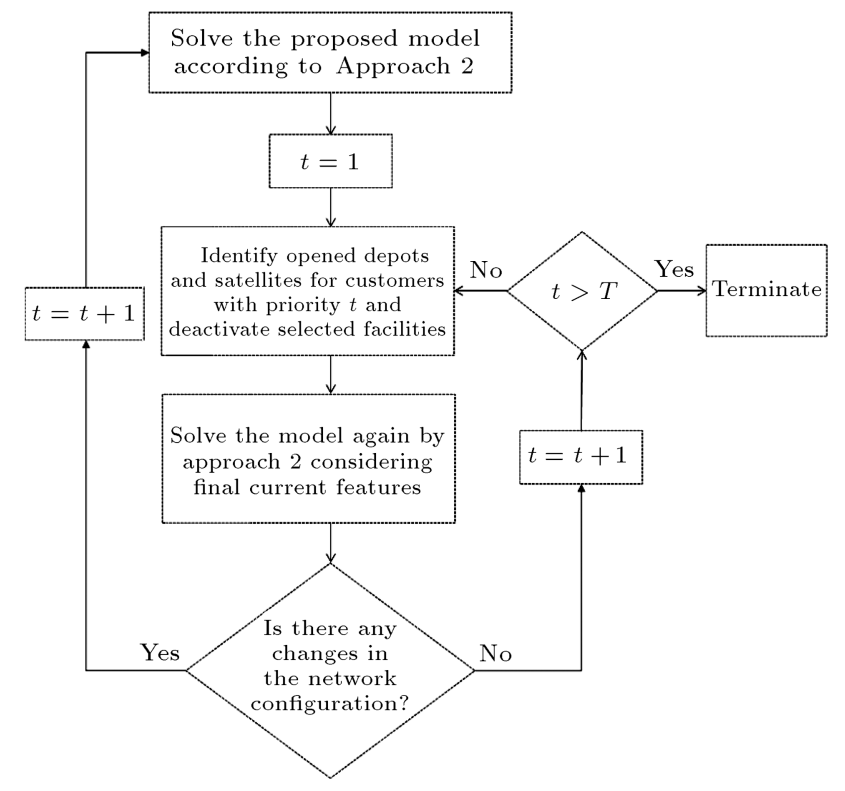

Figure 4. Flowchart of Approach 3.

\subsection{Approach 3 (attention to both total cost and customers' priorities)}

In this approach, the designer is interested in reducing the total system cost while he/she attempts to pay attention to the priorities of customer. The overall algorithm can be described as follows and its flowchart is depicted in Figure 4.

First, model 1 is solved, which results in obtaining the optimal establishment of depots and satellites. Then, the model is modified to improve transportation cost according to Approach 2. Afterwards, for each priority of customer, the opened facilities are determined and deactivated for improving the total cost. Then, the network is optimized again by Approach 2 considering the existing opened facilities. The mentioned approaches have different characteristics which have been compared in Table 1.

\section{Illustrative example}

In this section, a numerical hypothetical example of a distribution network is generated, randomly, in order to show efficiency and applicability of the proposed mathematical model. In the presented example, there are 3 potential depots, 3 satellites, and 4 customers.

Table 1. Comparison between illustrated approaches considering the importance of different factors.

\begin{tabular}{lcc}
\hline & $\begin{array}{c}\text { Customers' } \\
\text { priority }\end{array}$ & $\begin{array}{c}\text { Total cost } \\
\text { minimization }\end{array}$ \\
\hline Classical approach & Low & Very high \\
Approach 1 & Very high & Low \\
Approach 2 & High & Median \\
Approach 3 & Median & High \\
\hline
\end{tabular}

Other information of the example is reported in Table 2 .

In order to compare efficiencies of the proposed models with classical approach, first, we consider the customers without any priority. It is clear that if we consider the same priority for all customers, the results of the proposed approach confirm the classic one. So, the problem was solved by considering customers with equal priorities to be compared with the result of the proposed model.

By solving the proposed model according to Approach 1 in iteration $1(t=1)$, the results show that depot 1 and satellite 1 should be established. Then, this model is replicated again until all customers are being served (until $t=3$ ). For example, in iteration 3 , depot 3 and satellite 3 should be established to satisfy the 3rd customer's demand. Solving the model by using the second approach shows that depots 1,3 , and satellites 1, 3 are established. The result of Approach 2 implies that in order to serve the customer with priority 2 , it is affordable that satellite 3 will be established. Furthermore, considering the example by Approach 3 shows that since depot 3 is established in iteration 3 , the problem should be resolved according to Approach 2 , with consideration of the opened facilities. The distribution network configurations resulted by all the mentioned approaches have been compared in Table 3 and their final configurations have been illustrated in Figure 5.

Table 3 shows that the cost related to a customer with higher priority will be the lowest one, which

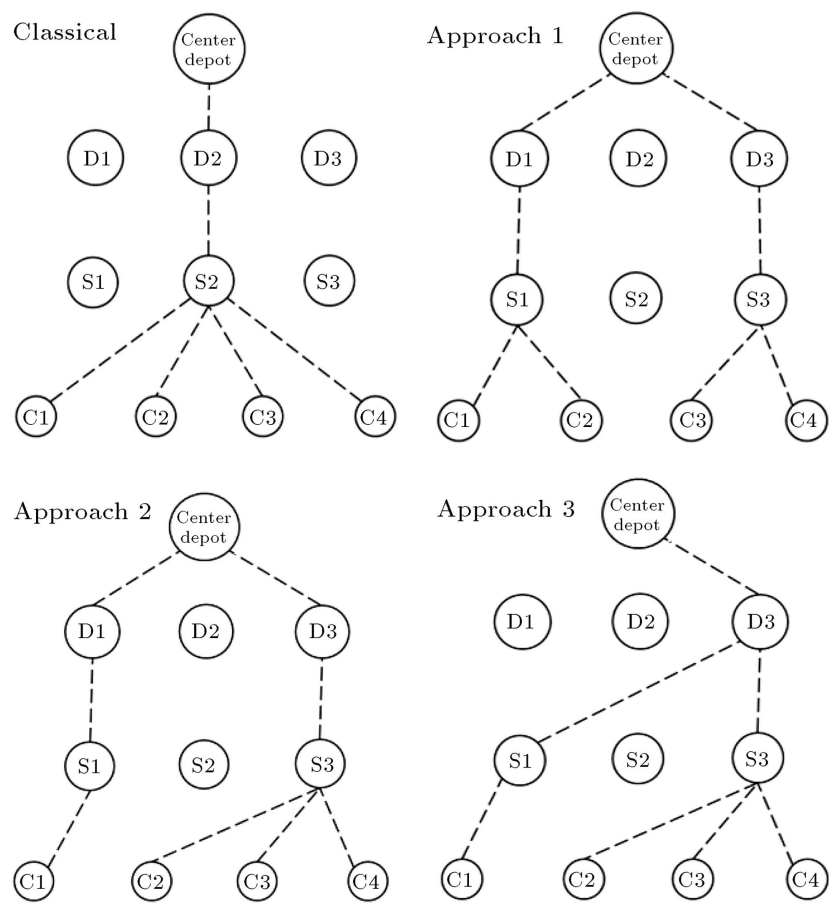

Figure 5. The distribution network configurations resulted by all the mentioned approaches. 
Table 2. Parameters of the hypothetical example.

\begin{tabular}{|c|c|c|c|c|c|c|c|c|c|c|}
\hline & \multicolumn{3}{|c|}{ Depot } & \multicolumn{3}{|c|}{ Satellite } & \multicolumn{4}{|c|}{ Customer } \\
\hline & 1 & 2 & 3 & 1 & 2 & 3 & 1 & 2 & 3 & 4 \\
\hline Fixed cost & 5000 & 5000 & 5000 & 1200 & 1200 & 1200 & - & - & - & - \\
\hline $\begin{array}{c}\text { Number of } \\
\text { available trucks }\end{array}$ & 25 & 25 & 25 & - & - & - & - & - & - & - \\
\hline Operation cost & - & - & - & 20 & 20 & 20 & - & - & - & - \\
\hline Demand & - & - & - & - & - & - & 120 & 120 & 120 & 120 \\
\hline Priority & - & - & - & - & - & - & 1 & 2 & 3 & 3 \\
\hline
\end{tabular}

Table 3. Summary of results obtained by all the mentioned approaches, including related costs for each priority in the distribution network.

\begin{tabular}{lcccc}
\hline & $\begin{array}{r}\text { Classical } \\
\text { approach }\end{array}$ & $\begin{array}{c}\text { Approach } \\
\mathbf{1}\end{array}$ & $\begin{array}{c}\text { Approach } \\
\mathbf{2}\end{array}$ & $\begin{array}{c}\text { Approach } \\
\mathbf{3}\end{array}$ \\
\hline Total cost & 13460 & 18720 & 18420 & 13560 \\
Priority 1 (c1) & 8040 & 7160 & 7160 & 7300 \\
Priority 2 (c2) & 2080 & 2160 & 1960 & 1960 \\
Priority 3 (c3,c4) & 3340 & 9400 & 9300 & 4300 \\
\hline
\end{tabular}

Table 4. Cumulative costs of customers with priority 1 to priority $t$.

\begin{tabular}{lccccccc}
\hline Customers' demands & p1 & p2 & p3 & p4 & p5 & Model \\
\hline (a) $150 / 150 / 150 / 150 / 150$ & 18690 & 20015 & 26255 & 33870 & $\Downarrow$ & 42720 & Classical model \\
& 22480 & 25195 & 27925 & 34790 & 36920 & Proposed model \\
& & & & & & & \\
(b) $120 / 140 / 150 / 150 / 150$ & 18560 & 19735 & 25975 & $\Downarrow$ & 33590 & 42430 & Classical model \\
& 21200 & 23755 & 26485 & 30560 & 35950 & Proposed model \\
& & & & & & & \\
(c) $100 / 120 / 150 / 150 / 150$ & 18455 & 19335 & $\Downarrow$ & 25560 & 33190 & 42235 & Classical model \\
& 20315 & 22515 & 25230 & 29060 & 35585 & Proposed model \\
& & & & & & \\
(d) $15 / 120 / 150 / 150 / 150$ & 16585 & $\Downarrow$ & 18950 & 25175 & 28415 & 35295 & Classical model \\
& 16680 & 18865 & 21580 & 24310 & 30560 & Proposed model \\
\hline
\end{tabular}

$\Downarrow$ Shows the cross point position.

is obtained by Approaches 1 and 2. However, the total cost will be more by the mentioned approaches. It confirms that the proposed approaches pay more attention to the customer priorities.

\section{Sensitivity analysis}

Some simulated numerical examples were generated according to the problem assumptions; then, the proposed model was solved by Approach 2 for each generated data set. Analysis of results shows that the cost related to higher priority customers, obtained with the proposed approach, is less than the cost related to the same customer, obtained by the classic one. As illustrated in Figure 6, their difference decreases for customers with lower priorities. Then, in a cross point, we will reach the same values for both approaches, while after the cross point, the classical approach has less customer-related costs for customers with lower priorities. The cross point has been depicted in Figure 6 as well.

For more analysis, some examples were generated with different demands for each customer and their effect on position change of cross point was studied. The results have been reported in Table 4 . The cross point position changes according to the demand changes has been depicted in Figure 7 . It shows that by decreasing demand of customers with higher priorities, the cross point position will be shifted to the left.

As an additional analysis, another example was generated with 4 customer priorities and customers were divided in selected priorities. The proposed model 


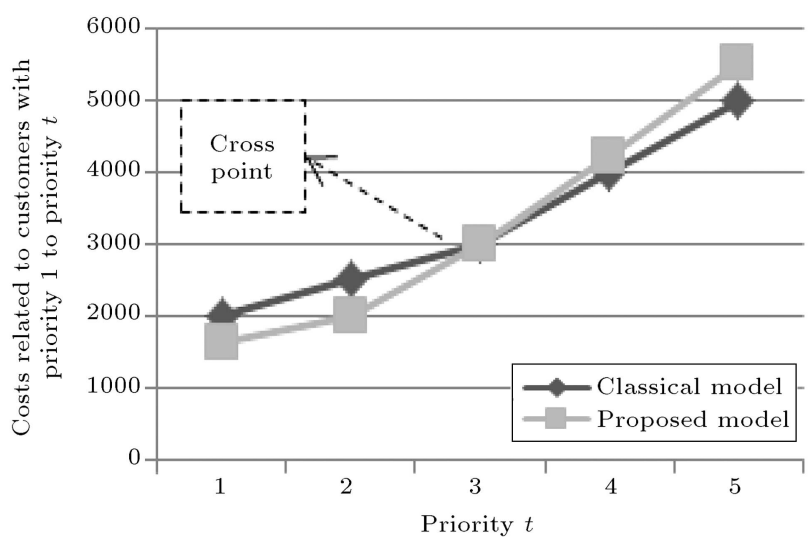

Figure 6. Illustration of cross point in a hypothetical example.

was solved for different numbers of customers. The analysis results show that by increase in the customers, the resulted total cost of the proposed model will be greater than that of the classical approach; however, they will converge when the number of customers increases to more than a specific number (in this example 15), as depicted in Figure 8.

On the other hand, when the priority of customers can be determined based on their demand value, by increase in the average of customers' demands difference, the proposed model and the classic one will have closer results to each other. This concept has been depicted in Figure 9.

\section{Conclusion}

In this paper, a distribution network design is investigated, considering a limited predefined number of trucks and priorities of customers. The present study verifies that the proposed model is affordable for

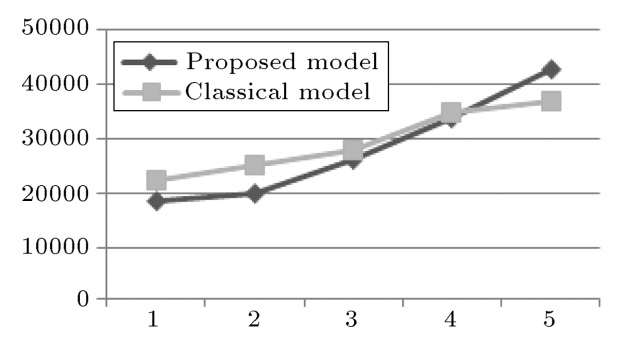

(a)

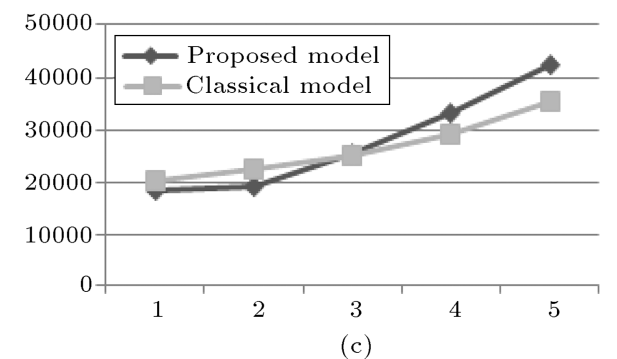

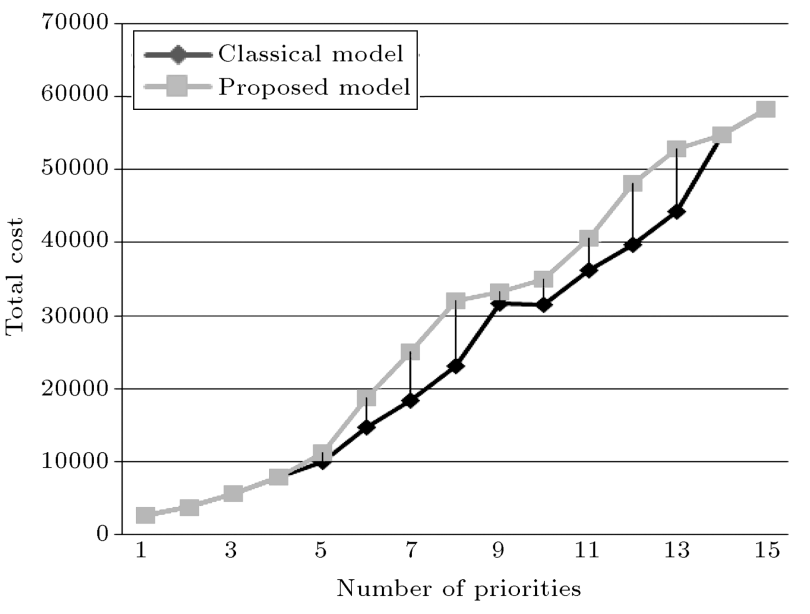

Figure 8. Total cost for different numbers of customers with predefined number of priorities.

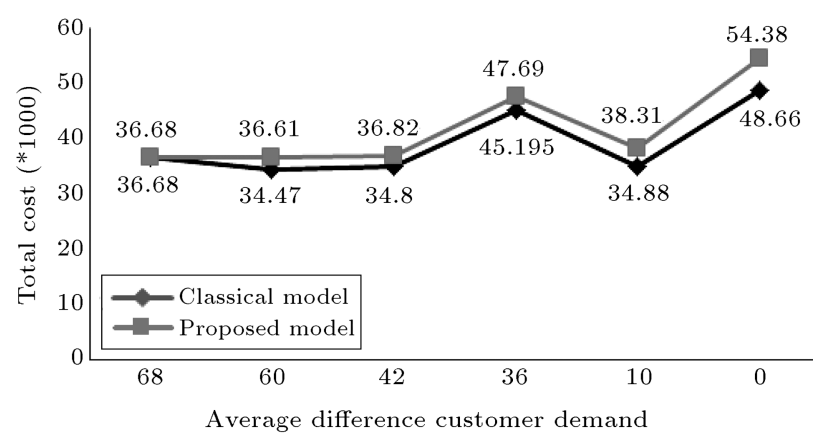

Figure 9. Effect of average customers' demands difference to the result of the proposed and classical models.

customers with higher priorities. We also present sensitivity analysis for demand of customers with higher priorities and changes of the cross point. The results confirm that the proposed approach can achieve the results of previous models by special values of param-

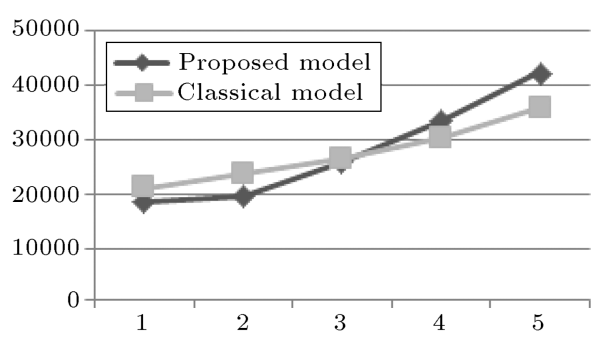

(b)

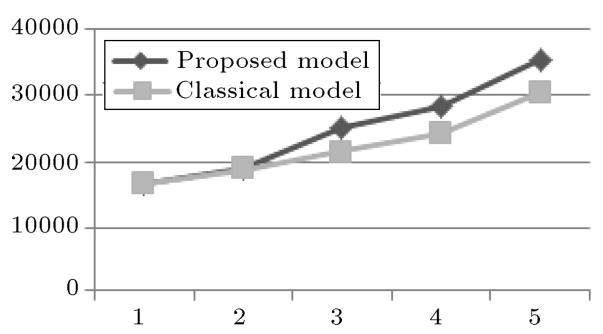

(d)

Figure 7. Cross point position change by change of customer demands. 
eters; however, the proposed approach can consider order priorities for customers. Study of a supply chain network design with different priorities of supply chain elements can be a field for future research. In addition, defining an explicit objective function for the customer priority in the multiple echelons DND problem and analyzing their quantitative tradeoff can be another valuable field for future study.

\section{References}

1. Noorul Haq, A. and Kannan, G. "Design of an integrated supplier selection and multi-echelon distribution inventory model in a built-to-order supply chain environment", International Journal of Production Research, 44(10), pp. 1963-1985 (2006).

2. Chopra, S. and Meindl, P., Supply Chain Management. Strategy, Planning \& Operation, in Das Summa Summarum des Management, C. Boersch and R. Elschen, Editors, Gabler, pp. 265-275 (2007).

3. Ambrosino, D. and Grazia Scutellà, M. "Distribution network design: New problems and related models", European Journal of Operational Research, 165(3), pp. 610-624 (2005).

4. Crainic, T.G. and Laporte, G. "Planning models for freight transportation", European Journal of Operational Research, 97(3), pp. 409-438 (1997).

5. Melo, M.T., Nickel, S. and Saldanha-da-Gama, F. "Facility location and supply chain management - A review", European Journal of Operational Research, 196(2), pp. 401-412 (2009).

6. Shen, Z.-J.M. and Daskin, M.S. "Trade-offs between customer service and cost in integrated supply chain design", Manufacturing \& Service Operations Management, $\mathbf{7}(3)$, pp. 188-207 (2005).

7. McLay, L.A. and Mayorga, M.E. "A model for optimally dispatching ambulances to emergency calls with classification errors in patient priorities", IIE Transactions, 45(1), pp. 1-24 (2012).

8. Melachrinoudis, E., Messac, A. and Min, H. "Consolidating a warehouse network: A physical programming approach", International Journal of Production Economics, 97(1), pp. 1-17 (2005).

9. Chan, F.T.S., Chung, S.H. and Wadhwa, S. "A hybrid genetic algorithm for production and distribution", Omega, 33(4), pp. 345-355 (2005).
10. Sapna Isotupa, K.P. and Samanta, S.K. "A continuous review inventory system with priority customers and arbitrarily distributed lead times", Mathematical and Computer Modelling, 57(5-6), pp. 1259-1269 (2013).

11. Tempelmeier, H. "Supply chain inventory optimization with two customer classes in discrete time", European Journal of Operational Research, 174(1), pp. 600-621 (2006).

12. Barbarosoglu, G. and Yazgac, T. "A decision support model for customer value assessment and supply quota allocation", Production Planning \& Control, 11(6), pp. 608-616 (2000).

13. Khaji, M.R. and Shafaei, R. "A system dynamics approach for strategic partnering in supply networks", International Journal of Computer Integrated Manufacturing, 24(2), pp. 106-125 (2011).

14. Gendron, B. and Semet, F. "Formulations and relaxations for a multi-echelon capacitated locationdistribution problem", Computers \& Operations Research, 36(5), pp. 1335-1355 (2009).

15. Goetschalckx, M., Supply Chain Engineering, Springer (2011).

16. Chen, D.-S., Batson, R.G. and Dang, Y., Applied Integer Programming: Modeling and Solution, John Wiley \& Sons (2001).

\section{Biographies}

Mahdi Bashiri is an Associate Professor of Industrial Engineering at Shahed University. He holds a BS degree in Industrial Engineering from Iran University of Science and Technology, and MS and PhD degrees in this field from Tarbiat Modares University. He is recipient of the 2013 Young National Top Scientist Award from Academy of Sciences of the Islamic Republic of Iran. His research interests are facilities planning, metaheuristics, and multi-response optimization.

Hamid Hasanzadeh received his BSc degree from Shahid Beheshti University in 2011. Now, he is an MS student of Industrial Engineering at Shahed University. His research interests are facility location, hub location problems, disaster management, and metaheuristic algorithms. 